\title{
PoPS: Mobile Access to Digital Library Resources
}

\author{
Nohema Castellanos \\ Universidad de las Américas, Puebla \\ Cholula, Puebla 72820 México \\ sp089077@mail.udlap.mx
}

\author{
J. Alfredo Sánchez \\ Universidad de las Américas, Puebla \\ Cholula, Puebla 72820 México \\ alfredo@mail.udlap.mx
}

\begin{abstract}
Mobile devices represent new opportunities for accessing digital libraries (DLs) but also pose a number of challenges given the diversity of their hardware and software features. We describe a framework aimed at facilitating the generation of interfaces for access to $D L$ resources from a wide range of mobile devices.
\end{abstract}

\section{Introduction}

In recent years there has been a widespread use of wireless devices. Analysts forecast that there will be more than a billion mobile phones in use within the next two years, and that over half of Internet accesses will be through non-PC devices. The main appeal of these appliances is mobility: the ability to access information and services anytime, anywhere [1].

Internet-enabled personal digital assistants (PDAs), cellular phones and a wide range of mobile devices represent a novel and promising facet to exploit digital library (DL) resources. However, wireless devices exhibit hardware and software features that are different from those in conventional computers. This makes it difficult to access DLs using the existing user interfaces and would make it necessary to redesign user interfaces for each kind of device.

We have designed a framework aimed to produce "generic" user interfaces for accessing DL resources from PDAs and cellular phones via personalizable Web environments. Our framework provides an efficient management of device resources as well as dynamic interfaces for appropriate deployment on small screens.

\section{Related work}

Several solutions have been proposed to generate Web pages that are well-suited to the limited screen and input capabilities of mobile devices. Methods to summarize and browse Web pages are presented in [3]. WAP (Wireless Access Protocol) includes tools to convert HTML into WML (Wireless Markup Language, the language used by WAP phones) [1]. An approach proposes XML-based markup languages to create generic interfaces: interfaces whose aspect may vary in different devices while their functionality remains the same in any of them [7]. Languages and tools that can be used to build generic interfaces include UIML (User Interface Markup Language) [4] and XUL (eXtensible User interface Language) [8]. These languages promote the separation of the interface description from the application as well as a scalable interface design.

An efficient management of device resources - such as CPU, memory, storage and bandwidth capabilities - is also crucial in mobile access. SS/CD (Small Screen/Composite Device) [9] and MOCA [2] are frameworks to provide mobile information services while efficiently managing device resources.

\section{Portable Personal Spaces (PoPS)}

As part of our digital libraries program, we have proposed the notion of personal spaces [10], which refers to virtual areas in a digital library through which users can manage DL resources and services, select contents relevant to their interests and organize them according to their individual needs and preferences. The concept of personal space has been instantiated successfully for use with conventional workstations. However, we would like to make our DL resources accessible to users from portable, wireless devices. Thus, we have designed a framework that provides developers with mechanisms for specifying interfaces for applications accessing DL resources to be rendered in various platforms, including portable and mobile devices. We have focused specifically on instantiating the concept of personal spaces using these facilities, hence we refer to our framework as "Portable Personal Spaces" (PoPS).

PoPS includes the notion of a unique personal space specification, which we name generic personal space. This specification is used to build interfaces for personal spaces that are suitable for each of the target devices in an automated fashion.

The framework consists of three major components: the generic personal space, a converter and an interface generator. The generic personal space contains interface descriptions written in a device-independent manner

Permission to make digital or hard copies of all or part of this work for personal or classroom use is granted without fee provided that copies are not made or distributed for profit or commercial advantage and that copies bear this notice and the full cit ation on the first page. To copy otherwise, to republish, to post on servers, or to redistribute to lists, requires prior specific permission and/or a fee. 
using an XML-syntax, which also enables the separation of content, logic and style. The converter transforms the interface descriptions into code written in the language used by the target device (taking device limitations into account). The interface generator builds the interfaces adapting the produced code to the preferences of the user.

\section{Implementation}

We have produced an implementation of PoPS that demonstrates our approach. So far, we have produced basic framework functionality and prototypical versions of personal spaces for PDAs and cellular phones.

The personal space version for PDAs was developed using the PersonalJava ${ }^{\mathrm{TM}}$ Runtime Environment for Windows CE Version 1.1 [6]. This prototype runs on an iPaq H3870 Pocket PC and accesses our DL via Internet. Figure 1 shows the interface of the main menu.

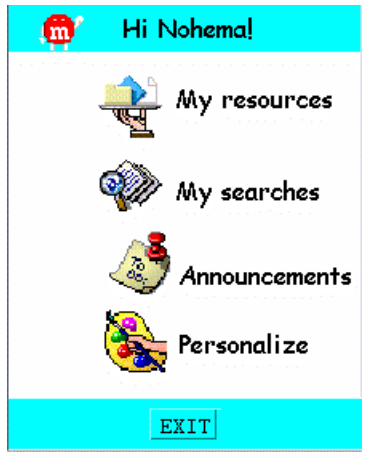

Figure 1. Interface of the main menu for PDAs

The PoPS specification to be rendered on cellular phones was developed in the Java ${ }^{\mathrm{TM}} 2$ Platform, Micro Edition $\left(\mathrm{J} 2 \mathrm{ME}^{\mathrm{TM}}\right)$ Wireless Toolkit 2.0 Beta 2 [5]. Our main testing environment has been an emulator that runs on a PC and accesses our DL via an intranet. The interface of the main menu is illustrated in Figure 2.

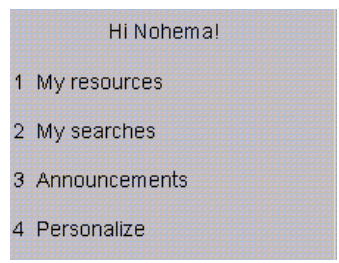

\section{Figure 2. Interface of the main menu for cellular phones}

\section{Preliminary results}

Our prototypical implementations have undergone usability tests with encouraging results. In general, test participants have adapted quickly to this kind of mouseless interaction and have been able to perform the tasks they intended or were asked to do. Most of them have found the interfaces attractive and have shown a great enthusiasm about the use of a mobile device to access our DL.

\section{Ongoing work}

Based on our usability test results, we are currently working on a formal specification of the generic personal space. After that, our work will focus on these stages: the development of the full-fledged interface generator, the implementation of the corresponding converter and the enhancement of the device resources manager.

\section{Acknowledgments}

This research is partly supported by the Mexican National Council of Science and Technology (CONACyT Projects 35804-A and G33009-A).

\section{References}

[1] Arehart, C., Professional Wap, Wrox Press, Birmingham UK, 2000.

[2] J. Beck, A. Gefflaut, and N. Islam, "MOCA: A Service Framework for Mobile Computing Devices", Proceedings of the ACM International Workshop on Data Engineering for Wireless and Mobile Access (MobiDE), Seattle USA, August 1999, pp. 62-68.

[3] O. Buyukkokten, H. Garcia-Molina, and A. Paepcke, "Seeing the Whole in Parts: Text Summarization for Web Browsing on Handheld Devices", Proceedings of the Tenth International Conference on World Wide Web (WWW10), Hong Kong, May 2001, pp. 652-662.

[4] Harmonia. Introduction to UIML. http://www.uiml.org.

[5] Java. http://java.sun.com/products/j2mewtoolkit.

[6] Java. http://java.sun.com/products/personaljava.

[7] O. Mayora-Ibarra, "Generation of Device Independent User Interfaces", Proceedings of International Workshop on Research \& Development of Human Communication Technologies for Conversational Interaction and Learning, Puebla México, 2002, pp. 1-3.

[8] Mozilla Org. Introduction to a XUL Document. http://www.mozilla.org/xpfe/xptoolkit/xulintro.html.

[9] T. Pham, G. Schneider, and G. Stuart, "A Situated Computing Framework for Mobile and Ubiquitous Multimedia Access using Small Screen and Composite Devices", Proceedings of the Eighth ACM International Conference on Multimedia, Marina del Rey CA, 2000, pp. 323-331.

[10] J.A. Sánchez, C. Proal, D. Pérez, and A. Carballo, "Personal and Group Spaces: Integrating Resources for Users of Digital Libraries", Proceedings of the $4^{\text {th }}$ Workshop on Human Factors in Computer Systems (IHC), Florianópolis Brazil, October 2001, pp. 15-17. 